Questions de communication

$24 \mid 2013$

Renouvellement des mises en scène télévisuelles de la politique

\title{
Marie-Anne PAVEAU, coord., «Texte, discours, interactions. Nouvelles épistémologies »
}

Semen. Revue de sémio-linguistique des textes et discours, 34, 2012, $202 \mathrm{p}$.

Jacques-Philippe Saint-Gerand

\section{OpenEdition}

\section{Journals}

Édition électronique

URL : http://journals.openedition.org/questionsdecommunication/8738

DOI : 10.4000/questionsdecommunication.8738

ISSN : 2259-8901

\section{Éditeur}

Presses universitaires de Lorraine

Édition imprimée

Date de publication : 31 décembre 2013

Pagination : 235-236

ISBN : 978-2-8143-0182-5

ISSN : 1633-5961

\section{Référence électronique}

Jacques-Philippe Saint-Gerand, « Marie-Anne paveau, coord., «Texte, discours, interactions. Nouvelles épistémologies » », Questions de communication [En ligne], 24 | 2013, mis en ligne le 01 février 2014 consulté le 22 septembre 2020. URL : http://journals.openedition.org/questionsdecommunication/ 8738 ; DOI : https://doi.org/10.4000/questionsdecommunication.8738 
Marie-Anne PaVeaU, coord., « Texte, discours, interactions. Nouvelles épistémologies ॥, Semen. Revue de sémio-linguistique des textes et discours, 34 . 2012,202 p.

La 34e livraison de Semen propose sept articles répartis en deux sections traitant de la question d'une révision critique des principes épistémologiques guidant la recherche en linguistique, plus spécifiquement en analyse des discours et en didactique interculturelle. La présentation du dossier thématique ainsi constitué par Marie-Anne Paveau donne à lire et comprendre les raisons de sa constitution. L'entrée dans une ère d'humanité augmentée par les nouvelles technologies de l'information et de la communication (TIC) oblige à prendre en considération le fait que la crise de densité des sciences humaines et sociales (SHS) nous a fait revenir sur les notions de dualisme, structuralisme et disciplinarité, que nous pouvons désormais envisager du point de vue d'observateurs ayant dépassé les binarismes fondateurs primaires. En effet, comme le montre globalement ce volume, les avancées du savoir se réalisent aujourd'hui non plus au cœur des disciplines, ce qui est particulièrement vrai pour les SHS, mais à leurs carrefours, ou plutôt dans l'échange et le dialogue entre les disciplines. Divisé en deux parties - « Disciplines et paradigmes 》 (pp. 19-70) et « Réalités, techniques, cultures 》 (pp. 7|-157) -, le présent volume offre ainsi un large panorama de cette épistémologie critique que réclame Marie-Anne Paveau dans son texte liminaire (pp. 7-17), c'est-à-dire cette « nécessité de (re)penser nos disciplines par rapport à des réalités évolutives, réalités tant des dispositifs théoriques et des postes d'observation des sciences humaines que du monde et des environnements désormais complexes qui le constituent » (p. 10). Lidée n'est pas nécessairement originale, mais, bien formulée et bien posée, elle permet d'envisager des développements substantiels. Ainsi, à l'heure du postdualisme, du poststructuralisme et de la postdisciplinarité, convient-il de songer à fonder « une intersdisciplinarité a-disciplinaire » tenant compte des acquis de la cognition dans l'approche des faits sociaux, et c'est là que les disciplines du texte, du discours et de l'interaction (TDI) peuvent aujourd'hui faire valoir leur intérêt et leur force.

Dans la première partie, Emmanuelle Danblon - «La rhétorique ou l'art de pratiquer» (pp. 19-34) revient sur la rhétorique comme mode de repenser l'épistémologie des disciplines des SHS en ce sens qu'elle permet « la mise en commun pratique et dynamique » (p. 32) des éléments de la raison qui sont le propre de l'homme. C'est encore la rhétorique que travaille Maria Zalęska - « Ordre et chaos dans les disciplines. L'exemple de la rhétorique » (pp. 35-50) pour montrer que « les conceptualisations sont des manières de se débrouiller sémiotiquement avec » ce qu'elle nomme le « chaordre » (p. 48), en d'autres termes « la chaotisation de l'ordre disciplinaire $[\ldots]$ regardée comme une source de créativité intellectuelle » (ibid.). II n'est pas certain que derrière ces néologismes s'affirment la puissance et la nouveauté d'une pensée stabilisée, mais l'effort est valeureux. Enfin, s'appuyant sur les travaux de Ron Scollon (1998, Mediated Discourse Analysis, Londres, Longman), Ingrid de Saint-Georges détaille de « Nouvelles épistémologies en analyse du discours et des interactions » (pp. 5l-69) qui reposent sur trois mutations actuellement en cours dans le secteur de l'étude des interactions: l'ouverture à la multimodalité, car l'étude des seuls énoncés verbaux est insuffisante pour traiter des interactions; la tagmémique de Kenneth L. Pike, en un sens, me semble-t-il, l'avait déjà fait sentir tout comme la praxémique de Robert Lafont le sous-entendait ; l'ouverture à l'histoire qui permet de recontextualiser les ensembles discursifs ; enfin, l'ouverture à l'expérience corporelle sans laquelle l'interaction demeure abstraite. C'est dans ce cadre que la théorie du discours médiatisé prend tout son sens en permettant de repenser « la contribution des realia, des médiations et des productions verbales.

Articulée autour de « Réalités, techniques, cultures 》 (pp. 7I-157), la seconde partie du volume s'ouvre sur la très stimulante et pertinente analyse de Guy AchardBayle consacrée à «Vérité-Réalité-Naturalité : la Relation et l'Ancrage » (pp.7l-93).Au terme de son analyse, ayant « parcouru le chemin du vérisme au naturalisme dans un sens puis dans l'autre » (p. 7I), l'auteur revient à John Searle et à son ouvrage de 1995 sur La construction de la réalité sociale (trad. de l'anglais par Claudine Tiercelin, Paris, Gallimard, 1998) qu'il rapproche judicieusement du texte fondateur du constructivisme sociologique de Peter Berger et Thomas Luckmann (La construction sociale de la réalité, Paris, A. Colin, 1996 [1966]) pour montrer combien le social est dépendant de l'humain. Ce qui l'autorise à voir le langage « comme source ou vecteur de l'humain dans l'évolution biologique » (p. 9l), et, partant, lui donne l'occasion de « réconcilier » les positions antagonistes que Michel de Fornel et Cyril Lemieux exposaient dans leur contribution syncrétique à Naturalisme versus Constructivisme? («Quel naturalisme pour les sciences sociales?»,pp.9-25, in: Michel de Fornel, Cyril Lemieux dirs, Naturalisme versus constructivisme? Paris, Éd. de l'EHESS, 2007). Revenant sur les rapports de la réalité et de la discursivité, Marie-Anne Paveau propose (p. 93) d'autres dimensions pour la théorie du discours puisqu'elle-même et Guy Achard-Bayle 
« ont en commun de considérer la réalité comme une donnée secondaire de la construction des discours ». En conséquence, elle propose de remettre en cause les grands dualismes (esprit/monde, intellect/affect, etc.) et note qu'aujourd'hui « les conceptions externalistes de l'esprit présentent ce dernier comme distribué dans les environnements »(p. 96). Ainsi, par exemple, les émotions. À cet égard, on s'étonne de ne pas trouver mention de la sémiotique des passions d'Algirdas Julien Greimas et Jacques Fontanille (Sémiotique des passions. Des états de choses aux états d'âme, Paris, Éd. Le Seuil, 1991). Lidée générale qui résulte de cette analyse est que le discours est essentiellement un matériau composite. Lui adresser la question du paramètre moral ( « la vertu [?] discursive ») comme critère d'évaluation du discours donne des arguments supplémentaires au besoin de décloisonner les disciplines, mais, à condition, bien sûr, de distinguer fermement entre « morale » et « éthique », ce qui déplace le problème à l'intérieur de la catégorie des discours. Dans une perspective « sciences de l'information et de la communication », Olivier Le Deuff - « Humanisme numérique et littératies » (pp. | 17-133) - revient sur la question de l'interaction homme-machine, le technohumanisme d'Ann Balsamo (« Technohumanism and the Rise of Digital Humanities », in : William Aspray, dir,, Digital Media : Technological and Social Challenges of the Interactive World, Lanham, Scarecrow Press, 201 I, pp. 213-226), pour poser le problème des rapports de l'humanisme numérique et des litteraties telles que Jack Goody les entend. Et l'auteur souligne très justement le danger de l'instrumentalisation qui résulte des formations (brevet informatique et internet - B2i -, certificat informatique et internet - C2i) pratiquées de nos jours, lesquelles ne permettent pas aux usagers de « comprendre les relations qui existent entre l'homme et les objets techniques » (p. 122), de sorte que ces derniers ne sont plus en mesure d'instrumenter le savoir afin de donner cet accès automatique à l'information raisonnée que devraient permettre les nouvelles technologies. D'où la nécessité de « définir les humanités numériques comme une transdiscipline » (p. 123) susceptible non seulement d'observer et penser le monde, mais aussi capable de l'améliorer et le faire changer (p. 132). Les questions que posent ces recherches sur de nouvelles épistémologies critiques trouvent une nouvelle dimension dans la contribution de Fred Dervin et Céline Tournebise «Rendez-vous ratés de l'interculturel en éducation? Une étude de cas de l'éducation à la communication interculturelle en Finlande » (pp. 135-157), qui fait état de l'éducation à la communication interculturelle en Finlande, car l'expérience internationale vécue apporte un supplément d'intérêt à la discussion d'objets qui peuvent souvent parâtre métathéoriques. En effet, la conclusion décevante de cette étude de cas montre bien que cette notion d'interculturalité demeure très floue, labile et instable dans l'esprit des enseignants finnois qui l'utilisent de sorte que « les spécialiste ne partagent pas toujours les mêmes discours, même s'ils utilisent les mêmes mots » (p. 152).

Mais, finalement, n'est-ce pas l'ensemble des recherches développées dans ce volume qui s'avère déceptif tant on peut avoir l'impression que la louable volonté de trouver des fondements épistémologiques sains et stables à l'analyse des productions discursives se heurte constamment aux leurres que proposent les constructions verbales des langues naturelles, tout à la fois objets d'étude dans les rapports qu'elles entretiennent avec le monde extérieur et formes obligées d'analyse de ces objets. En tout cas, le mérite de la livraison est d'offrir quantité d'arguments - de la rhétorique aux nouvelles technologies - pour penser ces rapports inédits. La rubrique «Varia 》 (pp. 15917I) donne à lire deux articles d'Alice Krieg-Planque (pp. 173-187, sur Denis Stoyanne, Petit glossaire de la guerre civile yougoslave, Lausanne, Éd. L'Âge d'homme, 1994) et de Stéphanie Kunert (« Dégenrer les codes, sémiotique du défigement », pp. |89-|9|), tandis que deux comptes rendus de lecture (pp. 193-198) - Alice Krieg-Planque, Caroline Ollivier-Yaniv, coords, « Les "petites phrases" en politique », Communication \& Langages, 168, juin 2011 et Émile Benveniste, Dernières leçons. Collège de France 1968 et 1969, Paris, Éd. de l'EhEss / Gallimard/Éd. Le Seuil, 2012) complètent l'organisation du volume.

Jacques-Philippe Saint-Gerand

CeReS, université de Limoges, F-87036 jacques-philippe.saint-gerand@unilim.fr

\section{Steven PINKER, L'instinct du langage.}

trad. de l'anglais (États-Unis) par Marie-France Desjeux, Paris, O. Jacob, 2013 [1994], 495 p.

II s'agit là de la réédition en format de poche du livre que ce chercheur canado-américain (Steven Pinker est né à Montréal) a publié il y a bientôt 20 ans... À l'époque de sa parution, l'ouvrage avait jeté quelque trouble dans la communauté scientifique, notamment chez les linguistes, les sociologues et même chez quelques psychologues en raison de la thèse innéiste ou nativiste que l'auteur soutenait en bon disciple des travaux de Noam Chomsky : « Le langage n'est pas un produit culturel qui s'apprend comme on apprend comment dire l'heure ou comment fonctionne le gouvernement de son pays. Au contraire, c'est une partie distincte de la structure biologique de notre cerveau. Le langage est 